\title{
Experimental investigation on flexural properties of FDM-processed PET-G specimen using response surface methodology
}

\author{
Nikolaos A. Fountas ${ }^{1}$, Ioannis Papantoniou ${ }^{2}$, John D. Kechagias ${ }^{3}$, Dimitrios E. Manolakos ${ }^{2}$ \\ and Nikolaos $M$. Vaxevanidis ${ }^{1 *}$ \\ ${ }^{1}$ Laboratory of Manufacturing Processes \& Machine Tools (LMProMaT), Department of Mechanical \\ Engineering Educators, School of Pedagogical and Technological Education (ASPETE), \\ Amarousion, GR 151 22, Greece \\ ${ }^{2}$ Manufacturing Technology Division, School of Mechanical Engineering, National Technical \\ University of Athens (NTUA), 9 Heroon Polytechniou Str., GR 15780 Athens, Greece \\ ${ }^{3}$ Design \& Manufacturing Lab., Department of FWSD, University of Thessaly, 11-13 V. Griva Str., \\ Karditsa, GR 4130, Greece
}

\begin{abstract}
The properties of fused deposition modeling (FDM) products exhibit strong dependence on process parameters which may be improved by setting suitable levels for parameters related to FDM. Anisotropic and brittle nature of 3D-printed components makes it essential to investigate the effect of FDM control parameters to different performance metrics related to resistance for improving strength of functional parts. In this work the flexural strength of polyethylene terephthalate glycol (PET-G) is examined under by altering the levels of different 3D-printing parameters such as layer height, infill density, deposition angle, printing speed and printing temperature. A response surface experiment was established having 27 experimental runs to obtain the results for flexural strength $(\mathrm{MPa})$ and to further investigate the effect of each control parameter on the response by studying the results using statistical analysis. The experiments were conducted as per the ASTM D790 standard. The regression model generated for flexural strength adequately explains the variation of FDM control parameters on flexural strength and thus, it can be implemented to find optimal parameter settings with the use of either an intelligent algorithm, or neural network.
\end{abstract}

\section{Introduction}

Fused deposition modelling is a widely known additive manufacturing method where the material in the form of filament is deposited in a "layer-by-layer" technique [1-4]. The material is deposited on a heated table with the aid of an extruder having a nozzle under high temperature and pressure. Printed components see services to both static and dynamic environments. In the case of dynamic environments, 3D-printed components may be

\footnotetext{
*Corresponding author: vaxev@aspete.gr
} 
subjected to different weather conditions or varying dynamic forces that may degrade their mechanical properties with emphasis to their strength. It is obvious that strength of $3 \mathrm{D}$ printed parts is greatly affected by the chemical composition of the material selected, as well as the control parameters of FDM method. As it occurs to any other manufacturing method, the settings for control parameters need to be carefully examined to end up with the best possible result from the perspective of low cost, productivity and strength. Based on the intended application, FDM parts are required to meet quality objectives such as dimensional accuracy, surface integrity, wear and mechanical strength.

It is reasonable that such objectives should be examined with reference to the effects of the independent process parameters. In [5] the dimensional accuracy of 3D-printed PLA material was examined by taking into account a number of input FDM parameters and the printing temperature suggested for PLA filaments. Compressive strength of standard geometries in FDM components has been examined in [6]. In [7] results were presented concerning the sliding wear behaviour for ABS material by examining the same experimental domain as in [6].

This study focuses on the experimental investigation of the effect of five important fused deposition modelling parameters, namely layer height, infill density; deposition angle, printing speed and printing temperature on the response of flexural strength (MPa) of PLA fabricated specimens designed as per the ASTM D790 standard. A response surface experiment corresponding to 27 runs was designed and conducted to obtain the results.

\section{Experimental}

This research focuses on examining the effect of five FDB control parameters on the flexural strength $(\mathrm{MPa})$ of PET-G specimens having standard geometry as per the ASTM D790. The parameters are determined as follows:

- Layer height: Layer height is the thickness of a layer deposited by the extruder's nozzle and is affected by the nozzle type.

- Infill density: The density of material used for filling interior regions of the part. Infill density is given as a percentage of a solid layer whose infill density is $100 \%$.

- Deposition/Orientation angle: The part's inclination angle on the printing bed, with reference to $\mathrm{X}$ and/or $\mathrm{Y}$ axes.

- $\quad$ Printing speed: The extruder's linear feed velocity.

- Printing Temperature: It is the temperature that the material is heated during the printing process. The recommended printing temperature for PET-G is $230-250{ }^{\circ} \mathrm{C}$.

The values corresponding to the levels of FDM parameters selected for the experiments were determined regarding the work material and general recommendations by the manufacturer of the equipment (3D printer and software) used. The FDM parameters for setting up the response surface experiment are presented in Table 1.

Table 1. Experimental input FDM parameters and corresponding levels.

\begin{tabular}{|l|l|l|l|}
\hline \multirow{2}{*}{ 3D printing parameters } & \multicolumn{3}{|c|}{ Levels } \\
\cline { 2 - 4 } & Low & Mid & High \\
\hline Layer height $(\mathrm{mm})$ & 0.1 & 0.2 & 0.3 \\
\hline Infill density $(\%)$ & 80 & 90 & 100 \\
\hline Deposition angle $(\mathrm{deg})$. & 0 & 45 & 90 \\
\hline Printing speed $(\mathrm{mm} / \mathrm{sec})$ & 40 & 50 & 60 \\
\hline Temperature $\left({ }^{\circ} \mathrm{C}\right)$ & 230 & 240 & 250 \\
\hline
\end{tabular}


The response surface experiment involved 27 runs. This design was selected to examine all possible interactions among the five FDM control parameters and establish a continuous experimental domain. The response surface design assists on studying the non-linear effect of all parameters to the response of flexural strength expressed through the stress (MPa) at severe deformation or total failure. Response surface design allows for fitting a second order polynomial regression relation among FDM parameters and the response of flexural strength and was preferred at that point of research owing to its ability of studying the curvature of parameter effects as well. The 3D CAD model corresponding to the flexural test specimen was designed as per the ASTM D790 standard in CATIA ${ }^{\circledR}$ V5 R18 and then exported as a *.STL file for importing it to Repetier Host ${ }^{\circledR}$ environment for modeling the printing process and slicing the part. Specimens were fabricated using the Craftbot ${ }^{\circledR}$ Plus Pro 3D printer. All tests are carried out at the temperature $23 \pm 2{ }^{\circ} \mathrm{C}$ and relative humidity $50 \pm 5 \%$ as per ISO R291:1977 (Plastics - Standard Atmospheres for Conditioning and Testing). As mentioned, the material used for fabricating the parts was polyethylene terephthalate glycol (PET-G) in the form of a $1.75 \mathrm{~mm}$ filament white coil. With reference to the experimental design each specimen was individually printed at the middle of the printer's working envelope to minimize the bed's positioning error effect. The temperature of the heated bed was kept at $65^{\circ} \mathrm{C}$. After fabrication, the specimens were dimensionally examined to ensure whether different geometric features are reasonably fabricated as per the nominal design. Flexural tests were conducted using an Instron ${ }^{\circledR} 4482$ dual-column Universal Testing Machine. Bluehill ${ }^{\circledR} 2$ environment was utilized to set up the interface such as the specimen dimension and other settings related to the overall process for performing the 3-point bending tests. During testing, the crosshead speed was maintained at $5 \mathrm{~mm} / \mathrm{min}$ for recording stable material deformation before failure. The crosshead motion continued until reaching a severe fracture or total fail of specimens. Experimental results involving flexure stress (MPa)-flexure strain (\%), as well as flexure load $(\mathrm{kN})$ - flexure extension $(\mathrm{mm})$ were collected and recorded through the data acquisition system of Bluehill® 2 environment. Fig.1a illustrates the experimental set-up, whereas Fig.1b illustrates the flexure load-extension / flexure stress-strain results from the 1st experimental run.

(a)

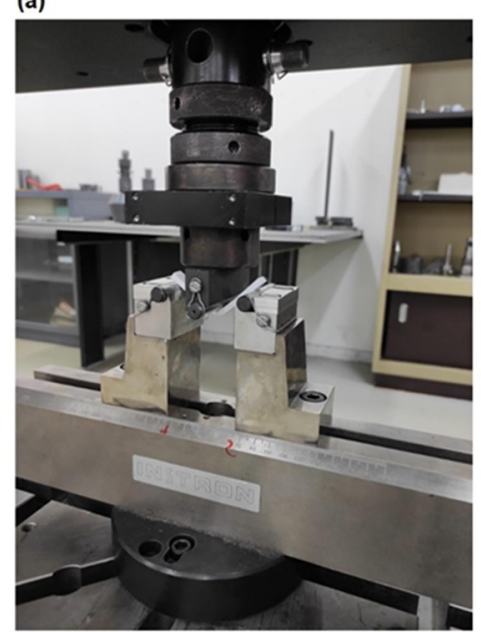

(b)
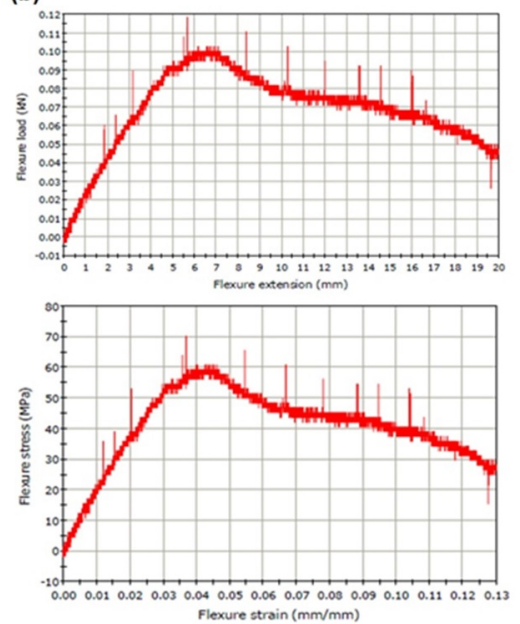

Fig. 1. (a) Instron ${ }^{(\mathbb{B}} 4482$ testing machine; (b) flexure test results for load (kN)-extension (mm) and stress (MPa)-strain. 
3-point bending tests were conducted as per the experimental design and maximum flexural stress-strain was recorded. The forces under which the specimens were subjected, indicate the point where the viscoelastic (recoverable) deformation is surpassed, leading thus to permanent (plastic) deformation. Different failure modes are observed in tested specimens fabricated with different input parameters. Specimens 1 and 2 were the only specimens that exhibited a brittle type of fracture that led to the total separation of the specimens. Fig. 2 presents the fractured surfaces of these specimens; Fig.2a for specimen 1 and Fig.2b for specimen 2 ; see also Table 2 which summarise the printing parameters of all tested items.
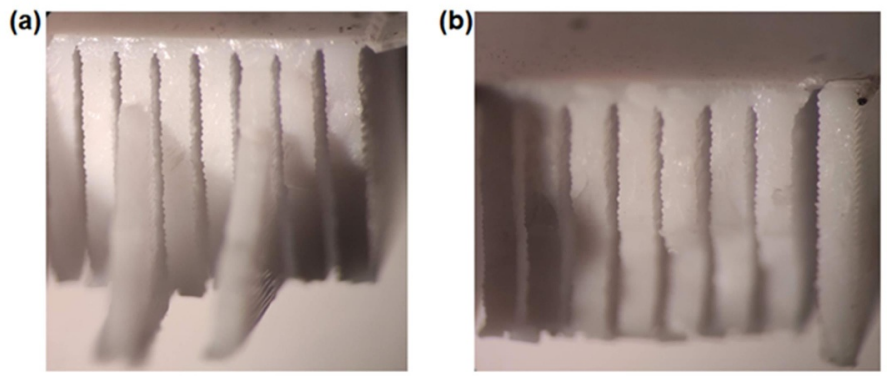

Fig. 2. Brittle type of fracture for deformed specimens: (a) specimen 1 and (b) specimen 2.

Specimens 10 to 12 as well as 25 to 27 presented only flexural plastic deformation. No external failure can be observed (e.g. cracks and delamination). Specimens 4 to 9,13 to 15 and 19 to 21 introduced flexural failure on the bottom side of the specimens. Flexural failure is caused by the tension load acting on the bottom side of the specimen, causing flexural cracks to form which eventually causes the failure of the specimen. Flexural cracks of specimen 15 are shown in Fig.3a. Specimens 1, 16 to 18 and 22 to 24 presented a more ductile type of flexural failure on the bottom side of the specimens. Furthermore, a delamination of the outer layers was also observed. Fig.3b and Fig.3c illustrate the ductile type of flexural failure of specimen 22 and the delamination of the outer layers of specimen 18 respectively.

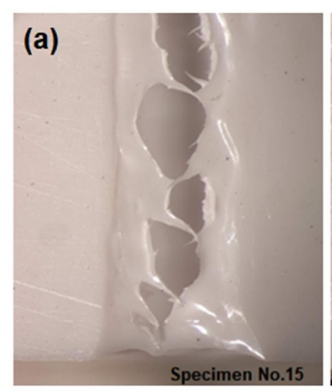

(c)

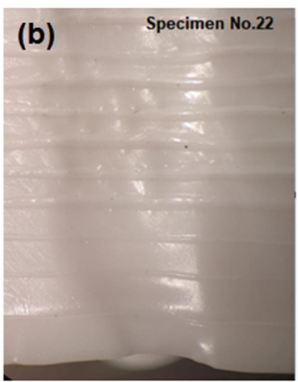

Specimen No.18

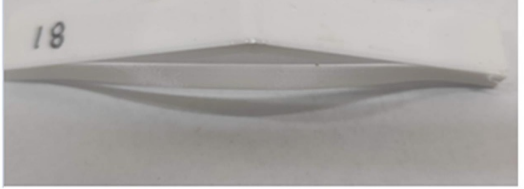

Fig. 3. (a) Flexural cracks of specimen 15; (b) The ductile type of flexural failure of specimen 22 and (c) Delamination effect of the outer layers for specimen 18 . 


\section{Statistical analysis and regression model generation}

Table 2 summarizes the design of experiments together with the results obtained in terms of maximum flexural stress $(\mathrm{MPa})$ for each experimental run. From the total of 27 experiments, experiments no. 16, 17 and 18 exhibited the highest flexural strength, i.e., $76.71,76.35$ and $78.18(\mathrm{MPa})$ respectively. This observation indicates that a layer height $0.2 \mathrm{~mm}$, an infill density $100 \%$, a deposition angle $0 \mathrm{deg}$. and a printing speed $50 \mathrm{~mm} / \mathrm{sec}$, are close to the most advantageous parameter levels in terms of PET-G material printing, under a temperature varying from $230^{\circ} \mathrm{C}$ to $250^{\circ} \mathrm{C}$.

Table 2. Series of response surface experiments and corresponding results.

\begin{tabular}{|l|c|c|c|c|c|r|}
\hline \multirow{2}{*}{$\begin{array}{l}\text { Exp. } \\
\text { no. }\end{array}$} & $\begin{array}{l}\text { Layer height } \\
(\mathrm{mm})\end{array}$ & $\begin{array}{c}\text { Infill density } \\
(\%)\end{array}$ & $\begin{array}{c}\text { Deposition } \\
\text { angle } \\
(\mathrm{deg})\end{array}$ & $\begin{array}{c}\text { Printing } \\
\text { speed } \\
(\mathrm{mm} / \mathrm{sec})\end{array}$ & $\begin{array}{c}\text { Temperature } \\
\left({ }^{\circ} \mathrm{C}\right)\end{array}$ & $\begin{array}{r}\text { Flexural } \\
\text { stress } \\
(\mathrm{MPa})\end{array}$ \\
\hline 1 & 0.1 & 80 & 0 & 40 & 230 & 59.1456 \\
\hline 2 & 0.1 & 80 & 0 & 40 & 240 & 61.9565 \\
\hline 3 & 0.1 & 80 & 0 & 40 & 250 & 64.0872 \\
\hline 4 & 0.1 & 90 & 45 & 50 & 230 & 32.2157 \\
\hline 5 & 0.1 & 90 & 45 & 50 & 240 & 34.7384 \\
\hline 6 & 0.1 & 90 & 45 & 50 & 250 & 31.3754 \\
\hline 7 & 0.1 & 100 & 90 & 60 & 230 & 33.6842 \\
\hline 8 & 0.1 & 100 & 90 & 60 & 240 & 40.4645 \\
\hline 9 & 0.1 & 100 & 90 & 60 & 250 & 61.9436 \\
\hline 10 & 0.2 & 80 & 45 & 60 & 230 & 32.7426 \\
\hline 11 & 0.2 & 80 & 45 & 60 & 240 & 34.6667 \\
\hline 12 & 0.2 & 80 & 45 & 60 & 250 & 32.4503 \\
\hline 13 & 0.2 & 90 & 90 & 40 & 230 & 38.4466 \\
\hline 14 & 0.2 & 90 & 90 & 40 & 240 & 44.6602 \\
\hline 15 & 0.2 & 90 & 90 & 40 & 250 & 37.0112 \\
\hline 16 & 0.2 & 100 & 0 & 50 & 230 & 76.7131 \\
\hline 17 & 0.2 & 100 & 0 & 50 & 240 & 76.3467 \\
\hline 18 & 0.2 & 100 & 0 & 50 & 250 & 78.1844 \\
\hline 19 & 0.3 & 80 & 90 & 50 & 230 & 33.7831 \\
\hline 20 & 0.3 & 80 & 90 & 50 & 240 & 38.3721 \\
\hline 21 & 0.3 & 80 & 90 & 50 & 250 & 34.7826 \\
\hline 22 & 0.3 & 90 & 0 & 60 & 230 & 62.1118 \\
\hline 23 & 0.3 & 90 & 0 & 60 & 240 & 64.2500 \\
\hline 24 & 0.3 & 90 & 0 & 60 & 250 & 65.5452 \\
\hline 25 & 0.3 & 100 & 45 & 40 & 230 & 65.9561 \\
\hline 26 & 0.3 & 100 & 45 & 40 & 240 & 67.0807 \\
\hline 27 & 0.3 & 100 & 45 & 40 & 250 & 68.1741 \\
\hline & & & & & & \\
\hline
\end{tabular}

Analysis of the experimental data obtained from the experimental design was carried out on MINITAB ${ }^{\circledR}$ R17 software using the full quadratic response surface model as given by Eq.1.

$$
y=\beta_{0}+\sum_{i=1}^{k} \beta_{i} x_{i}+\sum_{i=1}^{k} \beta_{i i} x_{i} x_{i}+\sum_{i<j} \sum \beta_{i j} x_{i} x_{j}
$$

$Y$ is the response of flexural strength $(\mathrm{MPa})$ and $x i$ is the $\mathrm{i}_{\text {th }}$ parameter. For significance check, F-value given in ANOVA table is used. Probability of F-value greater than calculated F-value due to noise is indicated by p-value. If p-value is less than 0.05 , significance of corresponding term is established. For lack of fit, $\mathrm{p}$-value should be greater 
than 0.05. An insignificant lack of fit is desirable because it indicates that any term excluded by the model is insignificant and that the developed model fits well. AndersonDarling normality test is used to verify the suitability of the model corresponding to the flexural strength for practical applications. If $\mathrm{p}$-value for the Anderson-Darling test is lower than the chosen significance level ( 0.05 in the current study), it is concluded that the data do not follow a normal distribution. In this research, ANOVA indicates that the quadratic model generated, is suitable for predicting the flexural strength of PET-G printed specimens with regression p-value less than 0.05 (i.e. 0.001 ) and a predicting error with $3.14 \%$ contribution. Based on p-value, it has been concluded that the flexural strength of PET-G specimens is mainly influenced by the linear terms and square terms followed by interaction terms, in general. Interactions among FDM parameters with strong effect on the response are $\mathrm{AxB}, \mathrm{AxC}$ and $\mathrm{BxC}$. The individual significance of each term is calculated by t-test at $95 \%$ confidence level, thus; terms having $p$-value less than 0.05 are significant. The coefficient of determination $\left(\mathrm{R}^{2}\right)$ which indicates the percentage of total variation in the response explained by the terms in the model has been found equal to $96.86 \%$. It is evident that all FDM parameters excluding printing temperature strongly affect the response of flexural strength (MPa). Results for the regression model are shown in Fig.4. Fig.4a illustrates a graphical comparison among experimental and predicted results. It can be observed that experiments no. 16,17 and 18 exhibited the highest result for flexural strength (MPa), as mentioned. Fig. $4 \mathrm{~b}$ verifies the model's adequacy in predicting the response. Since $p$-value of the normality plot is found to be far beyond 0.05 (i.e. 0.899 ) it indicates that residuals follow a normal distribution and predictions made by the regression model are in good agreement with experimental results.

(a)

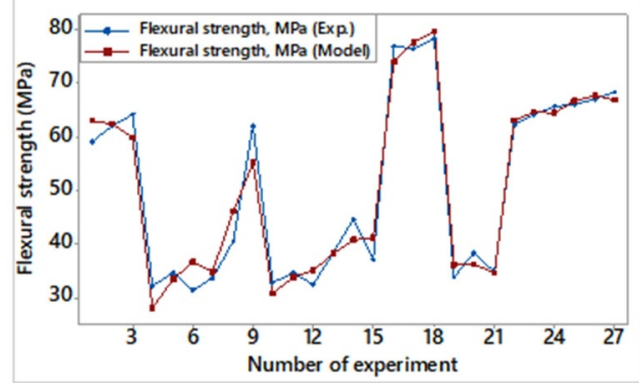

(b)

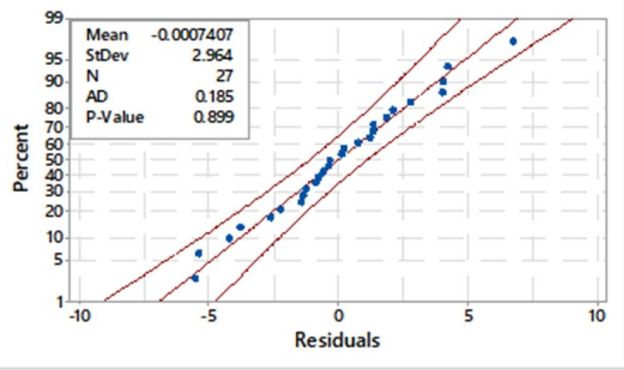

Fig. 4. (a) Experimental vs predicted results for flexural strength $(M P a)$; (b) probability plot of residuals at $95 \%$ c.i. - Anderson-Darling test.

By further examining ANOVA results that correspond to the interactions among FDM parameters it is observed that three interactions are critical and are Layer height $\mathrm{x}$ Infill density $(\mathrm{AxB})$, Layer height $\mathrm{x}$ Deposition angle $(\mathrm{AxC})$ and Infill density $\mathrm{x}$ Deposition angle $(\mathrm{BxC})$. The resulting surfaces from the analysis reveal the high complexity of the problem and the fact that parameters controlling the responses to engineering optimization problems should not be studied alone. Low layer height requires building a higher number of layers and this adds to the overall cost. In this experiment it is observed that when layer height is simultaneously examined with infill density, its level should be set to moderate values to increase strength, since the highest level will result to fewer deposited layers and this in turn will result to lower strength (Fig.5a). However, by setting a moderate level for layer height, infill density will maintain flexural strength by setting it to high levels, i.e., close to $100 \%$ (solid part). On the other hand, if it is decided to deposit many layers to build the part, (i.e. layer height equal to $0.1 \mathrm{~mm}$ ) then infill density should be set to lower values, i.e. 
$80 \%$ to maintain strength. In other words lower infill density compensates the number of layers required to have a strong structure. According to the indications observed in Fig.5b, high flexural strength can be maintained when layer height is set to its middle level, i.e. 0.2 $\mathrm{mm}$ and deposition angle is parallel to $X$ axis (i.e. deposition angle equal to $0 \mathrm{deg}$ ). Therefore the rectilinear $3 \mathrm{D}$ printing strategy parallel to $\mathrm{X}$ axis facilitates the component's endurance to withstand force, as the tension-compression effect occurs during 3-point bending experiment. The response surface depicted in Fig.5c shows that moderate-to-high deposition angles, i.e. 50-60deg., dramatically reduce flexural strength if infill density results to other than a solid structure. The resulting surface suggests that maximum flexural strength can be maintained by setting deposition angle to zero degrees while having a solid structure by setting $100 \%$ infill density. A good result for flexural strength can also be achieved by setting deposition angle parallel to $\mathrm{x}$-axis of the printing bed (deposition angle of 0 deg.) whilst setting $80 \%$ the infill density parameter.

(a) Layer height $\mathrm{x}$ Infill density

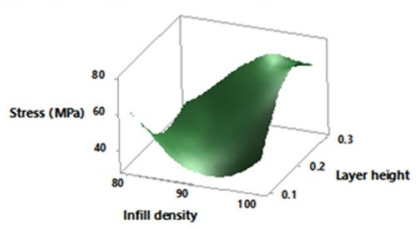

(b) Layer height $\times$ Deposition angle

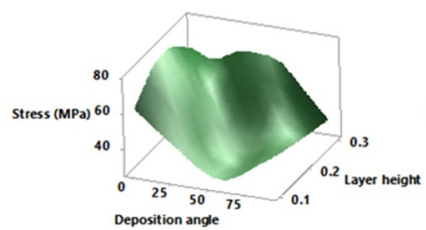

(c) Infill density $\times$ Deposition angle

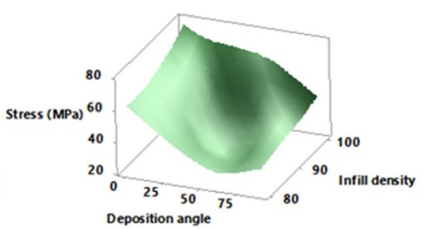

Fig. 5. Response surface plots for FDM parameter effects on flexural strength (MPa).

Other experimental observations involve the effect of printing speed on flexural strength. In general, when printing speed is set at low levels the thickness of the deposited filament is increased and may negatively affect the already deposited material in terms of deformation owing to stress accumulation. Consequently, the process may be prone to imperfections affecting the part's strength. According to the general experimental observations of this study, printing speed is suggested to be set at moderate-to-high levels to avoid deformation owing to high thermal energy concentration.

\section{Conclusions and future perspectives}

This work studied the effect of five control parameters for fabricating PET-G parts with fused deposition modelling (3D printing). The parameters were layer height, infill density, orientation angle, printing speed and printing speed. Experiments were conducted according to a response surface design with 27 runs to obtain results for flexural strength $(\mathrm{MPa})$. The response surface design allowed for the examination of the non-linear behaviour of FDM control parameters on flexural strength (MPa). Statistical analysis followed the experiments gave a regression model capable of explaining the experimental variability of parameters in the response and exploring the design space for further investigation. Owing to the complexity of the problem, the parameters were examined as interactions between two independent variables rather than single factors. Experimental observations as well as statistical outputs suggest that deposition angle is a dominant parameter to affect flexural strength of PET-G fabricated specimens. Deposition angle should be set parallel to x-axis of the printer's bed to withstand bending force and endure the tension-compression effect. Infill density is the second parameter that strongly affects flexural strength of PET-G. According to the results infill density should be set to high levels i.e., $100 \%$ to maintain strength but in this case a lower number of deposited layers should be determined (high layer height). If layer height is set to lower levels, infill density 
can be set nearly to $80 \%$. Printing speed strongly affects thermal energy and its absorption from neighbouring deposited layers. Printing speed should be set to middle and high levels to avoid thermal deformations of fabricated parts which in turn may negatively affect flexural strength. Nevertheless, printing speed and printing temperature do not seem to strongly affect the response of flexural strength.

Looking further ahead, more FDM materials will be evaluated and parameter optimization efforts will be conducted using artificial neural network models or intelligent algorithms. The regression model developed for predicting the response of flexural strength may play the role of the objective function to optimize FDM process. If another objective is to be introduced, i.e. fabrication time or material consumption, a multi-objective problem will be solved using several algorithms for comparison. In addition more destructive tests and wear experiments will be also under question for investigating the effect of FDM parameters for different materials.

The authors acknowledge the financial support for disseminating this work from the Special Account for Research of ASPETE (ELKE) through the funding program "Strengthening ASPETE's research".

\section{References}

1. S.Z. Hervan, A. Altınkaynak, Z. Parlar. Proc. Inst. Mech. En. J: J. En. Tribol (2020), doi: 1350650120966407.

2. S.K. Nayak, A. Satapathy. Proc. Inst. Mech. En. J: J. En. Tribol. 234 (2020)

3. E. Osuch-Słomka, R. Ruta, Z. Słomka. Proc. Inst. Mech. En. J: J. En. Tribol. 227 (2013)

4. N. Anjum, B. Suresha, S.L. Prasad. Tribol. Ind. 42 (2020)

5. K-E Aslani, K Kitsakis, JD Kechagias. SN Appl. Sci. 2 (2020)

6. A.K. Sood, R.K. Ohdar, S.S. Mahapatra. J. Adv. Res. 3 (2012)

7. Sood AK, Equbal A, Toppo V. CIRP J. Manuf. Sci. Technol. 5 (2012) 